\title{
THE MAY-WIGNER STABILITY THEOREM FOR CONNECTED MATRICES
}

BY HAROLD M. HASTINGS ${ }^{1}$

In 1972 R. M. May [4], following empirical results of M. R. Gardner and W. R. Ashby [2], sketched a proof of the following asymptotic stability theorem for large random linear differential systems:

$$
d x / d t=A x .
$$

May used deep results of E. P. Wigner [7], the "semicircle law" for eigenvalues of random symmetric matrices; see also Mehta [6].

MAY-Wigner StABIlity TheOREM. Let $B$ be an $n \times n$ matrix with $n^{2} C(0<C<1)$ randomly located nonzero entries, each chosen independently from a symmetric distribution with variance $\alpha^{2}$. Let $A=B-I$, and let $P(\alpha, n, C)$ be the probability that the corresponding differential system (1) has a stable equilibrium at 0 . Let $\epsilon>0$. Then $P(\alpha, n, C) \rightarrow 1$ as $n \rightarrow \infty$ provided $\alpha^{2} n C<1-\epsilon$; conversely, $P(\alpha, n, C) \rightarrow 0$ as $n \rightarrow \infty$ for $\alpha^{2} n C>1+\epsilon$.

This result provided a basis for studying the stability of neutral models in both cybernetics [2] and ecology (May [5] and references therein). Unfortunately Wigner's results are extremely complex, and may not apply to all random matrices (G. Sugihara, private communication, see also [6, p. 150]). We therefore sought a conceptually simpler proof.

We announce here a direct proof for matrices with connected underlying graphs. More precisely, the underlying graph of $A$ (with one edge joining $i$ and $j$ if $A_{i j}$ or $A_{j i}$ is nonzero) is asymptotically almost surely connected if

$$
C \geqslant(1+\epsilon) \log n / n,
$$

and asymptotically almost surely not connected if

$$
C \leqslant(1-\epsilon) \log n / n
$$

for any fixed positive $\epsilon$, (Bollobás [1, p. 143]). We assume the former condition holds; in particular the theorem holds for any constant $C$.

Received by the editors May 26, 1982.

${ }^{1}$ Partially supported by NSF grant MCS8102053.

1980 Mathematics Subject Classification. Primary 15A52, 34D05, 82A99, 92A15, $92 \mathrm{A17}$. 
There are three main steps in the proof. First, the columns of $\boldsymbol{A}$ approach orthogonality rapidly as $n \rightarrow \infty$. Secondly, for $\alpha^{2} n C<1-\epsilon, \epsilon$ fixed and positive, each column has $\mathcal{L}^{2}$ norm asymptotically almost surely less than $1-3 \epsilon^{2} / 4$; a suitable converse bound also holds $\alpha^{2} n C>1+\epsilon$. Finally, the Gerschgorin bound on the largest eigenvalue of a matrix is readily extended to a $l^{2}$ analogue for matrices with orthogonal columns.

A detailed proof and ecological applications and extensions will appear elsewhere [3]. We thank Drs. R. M. May and G. Sugihara for helpful conversations.

\section{REFERENCES}

1. B. Bollobás, Graph theory, Graduate Texts in Math., vol. 63, Springer, New York, 1979.

2. M. R. Gardner and W. R. Ashby, Connectance of large dynamic (cybernetic) systems: critical values for stability, Nature 228 (1970), 784.

3. H. M. Hastings, The May-Wigner stability theorem, J. Theoret. Biology (to appear).

4. R. M. May, Will a large complex system be stable?, Nature 238 (1972), 413-414. Princeton, N. J., 1974.

5. - Stability and complexity of model ecosystems, Princeton Univ. Press,

6. M. L. Mehta, Random matrices and the statistical theory of energy levels, Academic Press, New York and London, 1967.

7. E. P. Wigner, Statistical properties of real symmetric matrices with many dimensions, Proc. Fourth Canad. Math. Congr. (M. S. MacPhail, ed.) Univ. Toronto Press, Toronto, 1959, pp. $174-184$.

DEPARTMENT OF MATHEMATICS, HOFSTRA UNIVERSITY, HEMPSTEAD, NEW YORK 11550 